\title{
Thermal stability for domain wall mediated magnetization reversal in perpendicular STT MRAM cells with $\mathrm{W}$ insertion layers
}

\author{
G. Mihajlović, ${ }^{1, \text { a) }}$ N. Smith, ${ }^{1}$, b) T. Santos, ${ }^{1} \mathrm{~J}^{\mathrm{L}} \mathrm{Li},{ }^{1}$ B. D. Terris, ${ }^{1}$ and J. A. Katine ${ }^{1}$ \\ Western Digital Research Center, Western Digital Corporation, San Jose, CA 95119
}

(Dated: 5 August 2020)

\begin{abstract}
We present an analytical model for calculating energy barrier for the magnetic field-driven domain wall-mediated magnetization reversal of a magneto-resistive random access memory (MRAM) cell and apply it to study thermal stability factor $\Delta$ for various thicknesses of W layers inserted into the free layer (FL) as a function of the cell size and temperature. We find that, by increasing $\mathrm{W}$ thickness, the effective perpendicular magnetic anisotropy (PMA) energy density of the FL film monotonically increases, but at the same time, $\Delta$ of the cell mainly decreases. Our analysis shows that, in addition to saturation magnetization $M_{s}$ and exchange stiffness constant $A_{\text {ex }}$ of the FL film, the parameter that quantifies the $\Delta$ of the cell is its coercive field $H_{c}$, rather than the net PMA field $H_{k}$ of the FL film comprising the cell.
\end{abstract}

Thermal stability factor $\Delta$ quantifies retention of the spin-transfer-torque magneto-resistive random access memory (STT MRAM) cell. It is defined as the ratio of the energy barrier $E_{b}$ for magnetization $M$ reversal of the free layer (FL) of the magnetic tunnel junction (MTJ) comprising the memory cell, and the thermal energy $k_{B} T$, i.e. $\Delta=E_{b} /\left(k_{B} T\right)$ ( $k_{B}$ is the Boltzmann constant and $T$ is temperature). For a required small memory chip bit error rate BER $\ll 1$ against thermal bit flip $\frac{112}{10}$ and a retention time $t, \Delta_{\text {eff }}>\ln \left(f_{0} t /\right.$ BER $)$ is required, where $f_{0}=1 \mathrm{GHz}$ is the attempt frequency and $\Delta_{\text {eff }}=\Delta_{m}-\sigma^{2} / 2$ is the effective $\Delta$ value for the memory chip 3 . The latter expression assumes normal distribution of $\Delta$ values of individual chip cells, with $\Delta_{m}$ and $\sigma$ being the median and the standard deviation of the distribution. While the direct way to evaluate $\Delta_{\text {eff }}$ is by examining fraction of bits flipping their $M \mathrm{~s}$ as a function of $t$ at various $T \mathrm{~s}$ (the socalled retention bake method) ${ }^{3}$, this approach is rarely used as it is time consuming. Among many various alternative techniques ${ }^{4}$, Thomas et al ${ }^{[5}$ showed that, for the MTJ diameters $D>55 \mathrm{~nm}, \Delta_{m}$ and $\sigma$ values can also be determined by fitting the magnetic field $H$ induced empirical switching probabilitiy distributions $P(H)$ of individual cells to a Neel-Brown relaxation model ${ }^{1}$, namely

$$
P(H)=1-f_{0} t \exp [-\Delta(H)] \equiv 1-f_{0} t \exp \left[-\frac{E_{b}(H)}{k_{B} T}\right],
$$

with $E_{b}(H)$ calculated assuming domain wall-mediated $M$ reversal (DWMR). Micro-magnetic 6 and atomistic ${ }^{7}$ simulations suggest that MTJ FL with perpendicular magnetic anisotropy (PMA) can prefer DWMR down to $D \approx 25 \mathrm{~nm}$. In that case, $E_{b, \mathrm{dw}}=D \varepsilon_{\mathrm{dw}} t_{\mathrm{FL}}$ where $t_{\mathrm{FL}}$ is the FL thickness and $\varepsilon_{\mathrm{dw}}=$ $\sqrt{8 M_{s} H_{k} A_{\mathrm{ex}}}$ is the DW energy density $\left(M_{s}\right.$ is saturation magnetization, $H_{k}$ is the net PMA field and $A_{\mathrm{ex}}$ is the exchange stiffness constant of the FL). Alternatively, $\varepsilon_{\mathrm{dw}}=4 \sqrt{K_{\mathrm{eff}} A_{\mathrm{ex}}}$ where $K_{\text {eff }}=M_{S} H_{k} / 2$ is the FL PMA volume energy density. Thus, for the DWMR, $\Delta$ is expected to depend on $A_{\mathrm{ex}}$ and $K_{\mathrm{eff}}$ (i.e. $M_{s}$ and $H_{k}$ ) of the FL film. In addition to sandwiching

\footnotetext{
a)Electronic mail: goran.mihajlovic@wdc.com

b) Electronic mail: neil.smith@wdc.com
}

the CoFeB-based FL between two $\mathrm{MgO}$ layers $\stackrel{89}{ }$, a common approach to increasing $H_{k}$ is by inserting a thin non-magnetic layer, typically $\mathrm{Ta} \mathrm{a}^{8 \mid 10}, \mathrm{Mc}^{11 \mid 12}$ or $\mathrm{W}^{10 \mid 13}$. Recently, however, it has been reported that such insertion layers (ILs) dilute magnetic moment of the FL which reduces $M_{s}$, resulting in poor thermal stability performance at higher operational $T s$. Another study found that W ILs result in reduction of zero- $T$ $A_{\text {ex }}$, suggesting potential disadvantage of such ILs for achieving high $\Delta$ of MRAM cells for DWMR ${ }^{15}$. Ultrathin $\mathrm{CoFeB}$ FLs that provide superior STT switching performance without utilizing heavy-metal ILs to promote PMA ${ }^{16}$ have also been reported. In all these studies, $\Delta$ values for MRAM cell for DWMR were estimated using $H_{k}$ values measured on the full FL film. However, a direct, device-level study of the physical parameters quantifying $\Delta$ for DWMR of the FLs at operationrelevant $T$ s has been lacking.

Here we present an experimental study of $P(H)$ for perpendicular MRAM cells fabricated from FL films having variable thickness of the W IL $t_{\mathrm{W}}$ at $T=30,85$, and $125^{\circ} \mathrm{C}$. We describe an analytical model for calculating $E_{b, \mathrm{dw}}$ which introduces correction to the droplet model previously used in literature $5|7| 17 \mid 18$. By fitting $P(H)$ using this model, we find that $\Delta$ of MRAM cells decreases with increasing $t_{\mathrm{W}}$, even though $H_{k}$ and PMA energy per unit area $K_{\text {eff }} t_{\mathrm{FL}}$ of the full FL film are increasing. We show that this is not only due to decreasing $M_{s}$ and $A_{\mathrm{ex}}$ with increasing $t_{\mathrm{W}}$, but also due to decreasing $H_{c}$ of the cell. We determine DW width $w_{\mathrm{dw}}$ in range of $11-17 \mathrm{~nm}$ for $T \mathrm{~s}$ in range $30-125^{\circ} \mathrm{C}$, with largest values at highest $T$. Our results and analysis provide valuable insights into physical factors important for achieving high MRAM cell $\Delta$ for technologically relevant cell sizes and operational $T$ s.

In the basic droplet model ${ }^{7 / 17 / 18}$, a zero-width domain wall inside a circular FL is taken to be a circular arc of radius $r$ forming a right-angle with the FL perimeter (see Fig. 1(a)). This model, however, predicts that $E_{b, \mathrm{dw}}(H)>0$ for all finite $H$, an unphysical feature that is removed by including a finite domain wall width $w_{\mathrm{dw}} 5$. The latter can be implemented by reducing the area of both domains via modulation of the wall position (see Fig. 1(b)). In Ref. [5], this was done by modulating length of the radial coordinate as $\Delta r \leftrightarrow \pm w_{\mathrm{dw}} / 2$, while maintaining the right-angle constraint. However, this approach is mathematically flawed, since position of the right/exterior end of the radial line $r$ (i.e. point $\mathrm{O}$ in Figs. 1(a-b)) will 


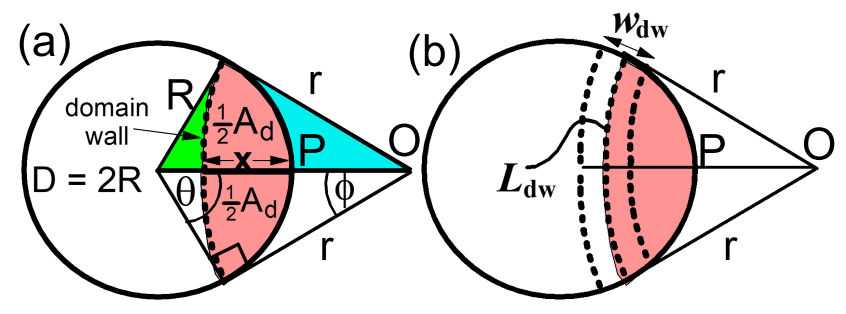

FIG. 1. Illustrations of (a) circular FL with reversed domain area $A_{d}$ formed at a distance $x$ from the right edge and (b) DW length $L_{\mathrm{dw}}$ and finite DW width $w_{\mathrm{dw}}$.

also be modulated. From Fig. 1, the correct identification is $\Delta x \leftrightarrow \pm w_{\mathrm{dw}} / 2$, with the right end of line $x$ fixed in position at the perimeter (point $\mathrm{P}$ in Figs. 1(a-b)). A more complete description of the required mathematical transformations can be found in Section I of the Supplementary Material. Defining $q \equiv x / R, \delta \equiv w_{\mathrm{dw}} / D$ and $q_{ \pm} \equiv q \pm \delta$, the results are:

$$
\theta(q)=\tan ^{-1}\left[\frac{q(1-q / 2)}{1-q}\right]
$$

$$
A_{d}(\theta)=\frac{D^{2}}{4}\left[\theta-\tan \theta+\left(\frac{\pi}{2}-\theta\right) \tan ^{2} \theta\right] \text {, }
$$

$$
L_{\mathrm{dw}}(\theta)=D\left(\frac{\pi}{2}-\theta\right) \tan \theta,
$$

$E(q)=L_{\mathrm{dw}}(q) \varepsilon_{\mathrm{dw}} t_{\mathrm{FL}}+|H| M_{s} t_{\mathrm{FL}}\left[\frac{D^{2} \pi}{4}-A_{d}\left(q_{+}\right)-A_{d}\left(q_{-}\right)\right]$

$$
E_{b, \mathrm{dw}}(H ; \delta) \cong E\left(q_{1}\right)-\frac{\pi}{4} D^{2} M_{s} H t_{\mathrm{FL}},
$$

$$
q_{1}=1+\varepsilon-\sqrt{1+\varepsilon^{2}},
$$

$$
\varepsilon=\frac{\varepsilon_{\mathrm{dw}}}{M_{s}|H| D} \text {. }
$$

The solutions (2e)- $(2 \mathrm{~g})$ for $E_{b, \mathrm{dw}}(H)$ are accurate to first order in $\delta$ and can be used to determine $\varepsilon_{\mathrm{dw}}$ and $w_{\mathrm{dw}}$ by fitting $P(H)$ using Eq. (1). A 2 nd order solution with $q_{1} \rightarrow q_{1}+\beta \delta^{2}$ is described in the Supplemental Material, though the difference is quite small in practical cases. By contrast, the $r$-based solution of Ref. [5] has leading error term of order $\delta$.

The MRAM film stacks used in this study consist of a Ta/Pt seed layer $(8.0 \mathrm{~nm}),(\mathrm{Co} / \mathrm{Pt}) / \mathrm{Ru} /(\mathrm{Co} / \mathrm{Pt}) / \mathrm{CoFeB}$ synthetic antiferromagnet reference layer (RL) $(7.1 \mathrm{~nm}), \mathrm{MgO}$ tunnel barrier, $\mathrm{CoFeB} / \mathrm{CoFe} / \mathrm{W} / \mathrm{CoFe} \mathrm{FL}, \mathrm{MgO}$ cap layer for enhancing $H_{k}$, and $\mathrm{Ru} / \mathrm{Ta}$ cap layer $(3 \mathrm{~nm})$. In the FL, the thickness of the magnetic layers was fixed at $1.5 \mathrm{~nm}$, while the thickness of the $\mathrm{W}$ spacer layer was varied, i.e. $t_{\mathrm{W}}=1.1,1.5,2.0$ and $2.6 \AA$. The films were deposited by magnetron sputtering in an Anelva C-7100 system and then annealed at $335^{\circ} \mathrm{C}$ for 1
TABLE I. Transport and magnetic properties of free layer films used in this study. The parameter values are expressed to the last significant digit based on the corresponding error analysis. $K_{i}$ is the intrinsic interfacial PMA surface energy density, physically independent of $M_{s}$, but here extracted from the values of $M_{s}$ and $H_{k}$ using the relation $K_{i}=M_{s} t_{\mathrm{FL}}\left(H_{k}+4 \pi M_{s}\right) / 2$.

\begin{tabular}{ccccccc}
\hline \hline $\begin{array}{c}t_{\mathrm{W}} \\
(\mathrm{A})\end{array}$ & $\begin{array}{c}\mathrm{RA} \\
\left(\Omega \mu \mathrm{m}^{2}\right)\end{array}$ & $\begin{array}{c}\text { TMR } \\
(\%)\end{array}$ & $\begin{array}{c}M_{\mathrm{S}} \\
\left(\mathrm{emu} / \mathrm{cm}^{3}\right)\end{array}$ & $\begin{array}{c}H_{\mathrm{k}} \\
(\mathrm{kOe})\end{array}$ & $\begin{array}{c}K_{\mathrm{eff}} t_{\mathrm{FL}} \\
\left(\mathrm{erg} / \mathrm{cm}^{2}\right)\end{array}$ & $\begin{array}{c}K_{i} \\
\left(\mathrm{erg} / \mathrm{cm}^{2}\right)\end{array}$ \\
\hline 1.1 & 11.2 & 135 & 1495 & 1.81 & 0.22 & 2.48 \\
1.5 & 11.1 & 142 & 1360 & 3.03 & 0.34 & 2.26 \\
2.0 & 11.1 & 141 & 1211 & 4.25 & 0.44 & 2.00 \\
2.6 & 11.2 & 134 & 1052 & 5.14 & 0.48 & 1.70 \\
\hline \hline
\end{tabular}

hour. The $\mathrm{MgO}$ layers were rf-sputtered from a $\mathrm{MgO}$ target. $\mathrm{RA} \cong 11 \Omega \mu \mathrm{m}^{2}$ and $\mathrm{TMR} \cong 140 \%$ values are measured on the annealed films by current-in-plane tunneling (CIPT) ${ }^{\sqrt{19}}$ at room $T$ (see Table I). $M_{S}$ was obtained by measuring the magnetic moment of the FL using vibrating sample magnetometry and dividing it with the FL volume assuming the full FL thickness $t_{\mathrm{FL}}$, including $t_{\mathrm{W}} . H_{k}$ of the FL was measured by full film ferromagnetic resonance. From Table I, one can see that while $M_{s}$ monotonically decreases with increasing $t_{W}, H_{k}$ and $K_{\text {eff }} t_{\mathrm{FL}}$ calculated using corresponding $M_{s}$ and $H_{k}$ values increase. However, $K_{i}$ decreases with increasing $t_{\mathrm{W}}$ (see the last column of Table I), implying that intrinsic interfacial PMA also decreases with increasing $t_{\mathrm{W}}$ and that the net increase in $H_{k}$ and $K_{\mathrm{eff}} t_{\mathrm{FL}}$ at room $T$ is solely due to reduction of the $M_{s}$ of the FL.

Circular MRAM test device cells are fabricated using $193 \mathrm{~nm}$ deep UV optical lithography, followed by reactive ion etching a hard mask, ion milling the MRAM film, $\mathrm{SiO}_{2}$ refill and chemical mechanical planarization. Electrical critical dimension CD of each device is determined using film-level RA provided in Table I and the device resistance in parallel state $R_{\mathrm{P}}$ as $\mathrm{CD}=\sqrt{4 \mathrm{RA} /\left(\pi R_{\mathrm{P}}\right)}$. CD values were clustered around four target sizes of $65,90,105$ and $120 \mathrm{~nm}$.

The $P(H)$ distributions are obtained by sweeping $H$ via a staircase ramp with the step of 5 Oe and with a dwell time of $0.2 \mathrm{~ms}$, and measuring the MTJ resistance $R$ at low bias voltage of $10 \mathrm{mV}$ to minimize STT effects on $M$ reversal. Fig. 2(a) shows an example of one hundred $R$ vs $H$ transfer loops measured at $T=30{ }^{\circ} \mathrm{C}$, for a device having $\mathrm{CD} \equiv D \cong 65 \mathrm{~nm}$, while the corresponding empirical $P(H)$ and fit to Eq (1) using $E_{b}(H)$ as expressed in Eqs. (2e)-(2g) is shown in Fig. 2(b). $\mathrm{P} \rightarrow \mathrm{AP}$ and $\mathrm{AP} \rightarrow \mathrm{P}$ branches are fit simultaneously by substituting $H \rightarrow H-H_{\text {offset }}$ with fixed $H_{\text {offset }}=H_{\mathrm{P} \rightarrow \mathrm{AP}}^{P=0.5}-H_{\mathrm{AP} \rightarrow \mathrm{P}}^{P=0.5}$ and fixed $M_{s}$, leaving $\varepsilon_{\mathrm{dw}}$ and $\delta$ as the only two fitting parameters. From here, $\Delta=D t_{\mathrm{FL}} \varepsilon_{\mathrm{dw}} / k_{B} T, w_{\mathrm{dw}}=D \delta$. For the device shown in Fig. 2(b) we obtained $w_{\mathrm{dw}}=12.7 \mathrm{~nm}$ and $\varepsilon_{\mathrm{dw}}=6.2 \mathrm{erg} / \mathrm{cm}^{2}$, corresponding to $\Delta=154$.

Fig. 3(a) shows $\varepsilon_{\mathrm{dw}}$, obtained by fitting $P(H)$ using Eqs. (1)-(2), as a function of $D$ at $T=30^{\circ} \mathrm{C}$ for FLs with different $t_{\mathrm{W}} \cdot \varepsilon_{\mathrm{dw}}$ increases for thinner $\mathrm{W}$, as well as for smaller $D$. The latter is quite moderate and can be attributed to mildly increasing $H_{k}$ with decreasing $D$ due to reduction of dipolar shape anisotropy. On the other hand, the dependence on $t_{\mathrm{W}}$ is 

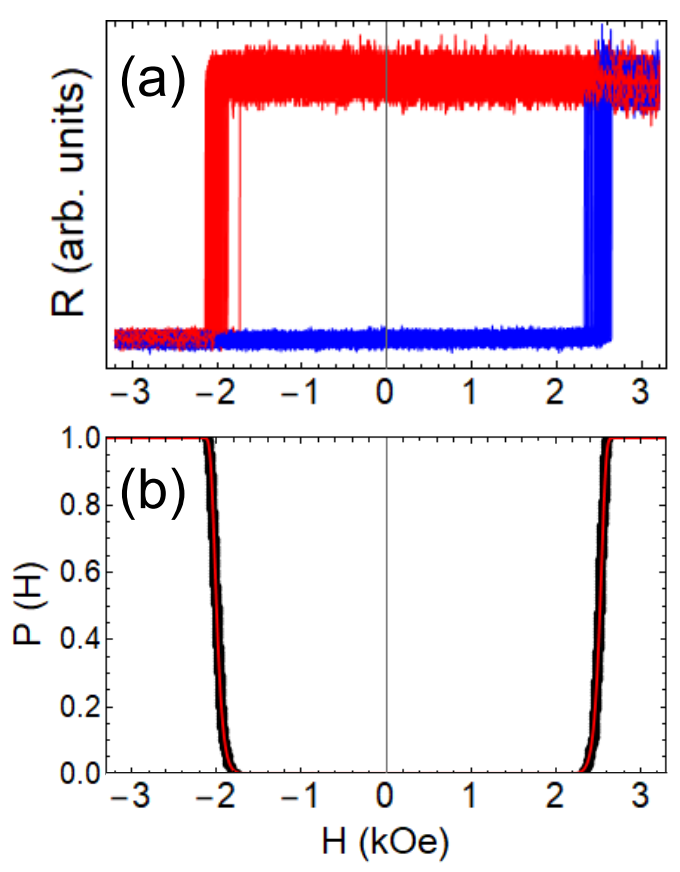

FIG. 2. (a) One hundred $R$ vs $H$ loops measured for a MRAM cell with $t_{\mathrm{W}}=1.1 \AA$ and $D=65 \mathrm{~nm}$ at $T=30{ }^{\circ} \mathrm{C}$. (b) Empirical $P(H)$ (black circles) corresponding to switching fields shown in part (a) of the Figure and fit to DWMR model (red lines) as described in text.

much stronger, resulting in about $30 \%$ increase of $\varepsilon_{\mathrm{dw}}$, from approximately $4.5 \mathrm{erg} / \mathrm{cm}^{2}$ for $t_{\mathrm{W}}=2.6 \AA$ to approximately $6 \mathrm{erg} / \mathrm{cm}^{2}$ for $t_{\mathrm{W}}=1.1 \AA$. This suggests that reduction of $H_{k}$ with decreasing $t_{\mathrm{W}}$ is more than compensated by increases in $M_{s}$ and, possibly, $A_{\mathrm{ex}}$.

Fig. 3(b) shows the corresponding $\Delta$ values as a function of $D . \Delta$ is highest for the FL with lowest $t_{\mathrm{W}}$, and lowest for the FL with highest $t_{\mathrm{W}}$. This is in direct opposition to the trends of $H_{k}$ and $K_{\text {eff }} t_{\mathrm{FL}}$ (see Table I). One can also see that while the difference in $\Delta$ values between thinnest and thickest W IL is significant, this difference is quite small between FLs with $1.1 \AA$ and $1.5 \AA \mathrm{W}$ IL. This suggests that for the given magnetic thickness and composition of the FL in our study, the optimal $t_{\mathrm{W}}$ resulting in maximum $\Delta$ is $\approx 1 \AA$.

Another parameter obtained directly by fitting $P(H)$ is $w_{\mathrm{dw}}$, shown in Fig. 3(c). We find $w_{\mathrm{dw}} \cong 11-15 \mathrm{~nm}$ for all $D$ and $t_{\mathrm{W}}$ values. These are smaller than reported previously for PMA thin films where $w_{\mathrm{dw}}$ was measured by magneto-optical Kerr microscopy imaging 2021 , but are consistent with device-level micromagnetic simulation results ${ }^{6}$. However, unlike $\varepsilon_{\mathrm{dw}}$ and $\Delta, w_{\mathrm{dw}}$ does not depend monotonically on $t_{\mathrm{W}}$ : it is largest for $t_{\mathrm{W}}=1.1 \AA$, smallest for $t_{\mathrm{W}}=1.5 \AA$, while the values for $t_{\mathrm{W}}=2.0 \AA$ and $2.6 \AA$ are in between.

The co-existence of highest $\varepsilon_{d w}$ and $w_{\mathrm{dw}}$ for thinnest $\mathrm{W}$, suggests largest $A_{e x}$ for this case, as both are $\propto \sqrt{A_{e x}}\left(w_{\mathrm{dw}}=\right.$ $2 \ln 2 \sqrt{A_{\mathrm{ex}} / K_{\mathrm{eff}}}$, see Section II of Supplementary Material for derivation of this expression). This is indeed the case, as can be seen in Fig. 3(d) where we plot calculated $A_{e x}=$ $\varepsilon_{\mathrm{dw}} w_{\mathrm{dw}} /(8 \ln 2)$. Indeed, $A_{\mathrm{ex}}$ is highest for thinnest $\mathrm{W}$ and decreases with increasing $\mathrm{W}$ thickness. This decrease, averaged
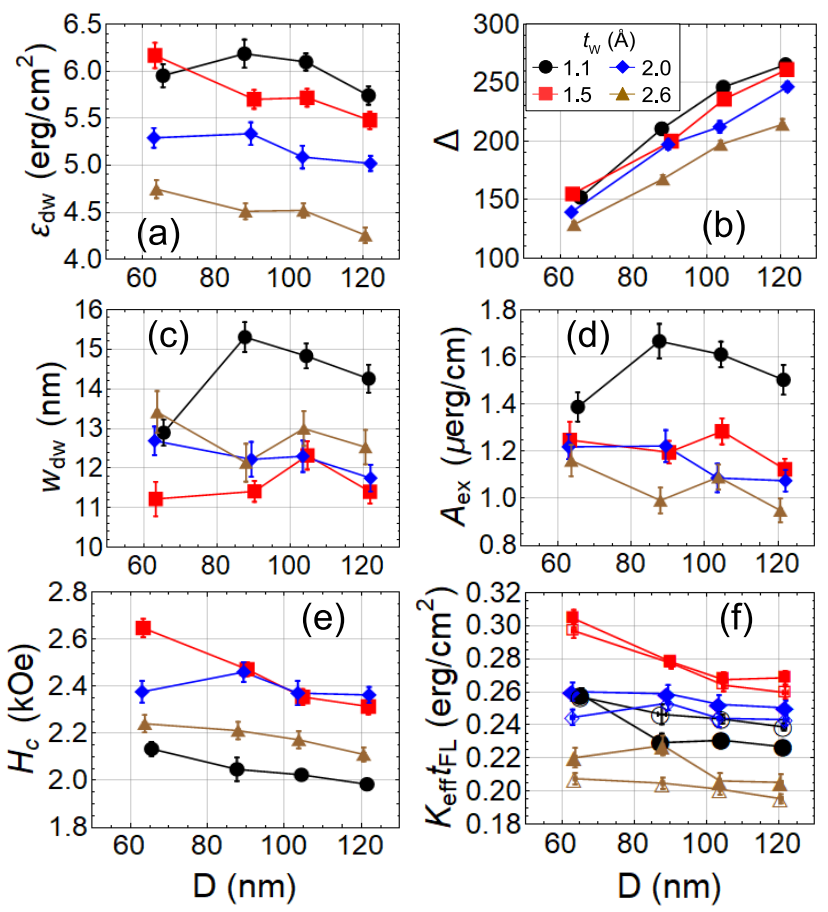

FIG. 3. (a) $\varepsilon_{\mathrm{dw}}$, (b) $w_{\mathrm{dw}}$, (c) $\Delta$, (d) $A_{\mathrm{ex}}$, (e) $H_{c}$ and (f) $K_{\mathrm{eff}} t_{\mathrm{FL}}=$ $(\ln 2 / 2)\left(\varepsilon_{\mathrm{dw}} / w_{\mathrm{dw}}\right) t_{\mathrm{FL}}$ (full symbols) and $K_{\mathrm{eff}} t_{\mathrm{FL}}=M_{s} H_{c} t_{\mathrm{FL}} / 2$ (open symbols) as a function of device diameter for FLs with various $t_{\mathrm{W}}$ determined by fitting $P(H)$ at $T=30{ }^{\circ} \mathrm{C}$ using DWMR model. Each data point corresponds to median and standard error from tens of studied MRAM cells of the same nominal size. The legend shown in part (b) of the Figure refers to other parts as well.

over all device sizes, is monotonic (see also Fig.4(e)). This is consistent with previously reported finding 5 15/21] although the values $A_{e x} \cong 1.0-1.7 \mu \mathrm{erg} / \mathrm{cm}$ that we obtain are approximately a factor of 2-3 higher compared to these reports. Thus, based on simple relation between $w_{\mathrm{dw}}, A_{\mathrm{ex}}$ and $K_{\mathrm{eff}}$, the nonmonotonic dependence of $w_{\mathrm{dw}}$ on $t_{\mathrm{W}}$ suggests non-monotonic dependence of $K_{\text {eff }}$ and likely $K_{\text {eff }} t_{\mathrm{FL}}$ in our fabricated cells, contrary to the results obtained from full films (see Table I). This is indeed the case, as can be seen in Fig. 3(f) where we plot $K_{\text {eff }} t_{\mathrm{FL}}=(\ln 2 / 2)\left(\varepsilon_{\mathrm{dw}} / w_{\mathrm{dw}}\right) t_{\mathrm{FL}}$ (full symbols). $K_{\mathrm{eff}} t_{\mathrm{FL}}$ increases when $t_{\mathrm{W}}$ is increased from $1.1 \AA$ to $1.5 \AA$, but it then decreases for larger $t_{\mathrm{W}}$. Surprisingly, the observed trend can be well reproduced by assuming that $K_{\text {eff }}$ in our devices is determined by device-level coercive field $H_{c}$ (see Fig. 3(e)) instead of the film level $H_{k}$, i.e. $K_{\text {eff }}=M_{s} H_{c} / 2$. The $K_{\text {eff }} t_{\mathrm{FL}}$ values calculated this way are shown in Fig. 3(f) as open symbols, and are in good quantitative as well as qualitative agreement with $K_{\text {eff }} t_{\mathrm{FL}}$ results from fitting to the DWMR model (full symbols in the same figure).

This finding suggests that $\Delta$ in devices, in the DWMR regime, is determined, in addition to the $M_{s}$ and $A_{\mathrm{ex}}$ of the FL film, by the device level $H_{c}$, which may, or may not be directly proportional to $H_{k}$ of the film (compare $H_{c}$ values in Fig. 3(e) and $H_{k}$ values in Table I). Our finding contrasts previous reports, where $K_{\text {eff }} t_{\mathrm{FL}}$ measured at the film level was used to either evaluate $A_{e x}$ from $\Delta$ measured on cells ${ }^{14}$, or to esti- 

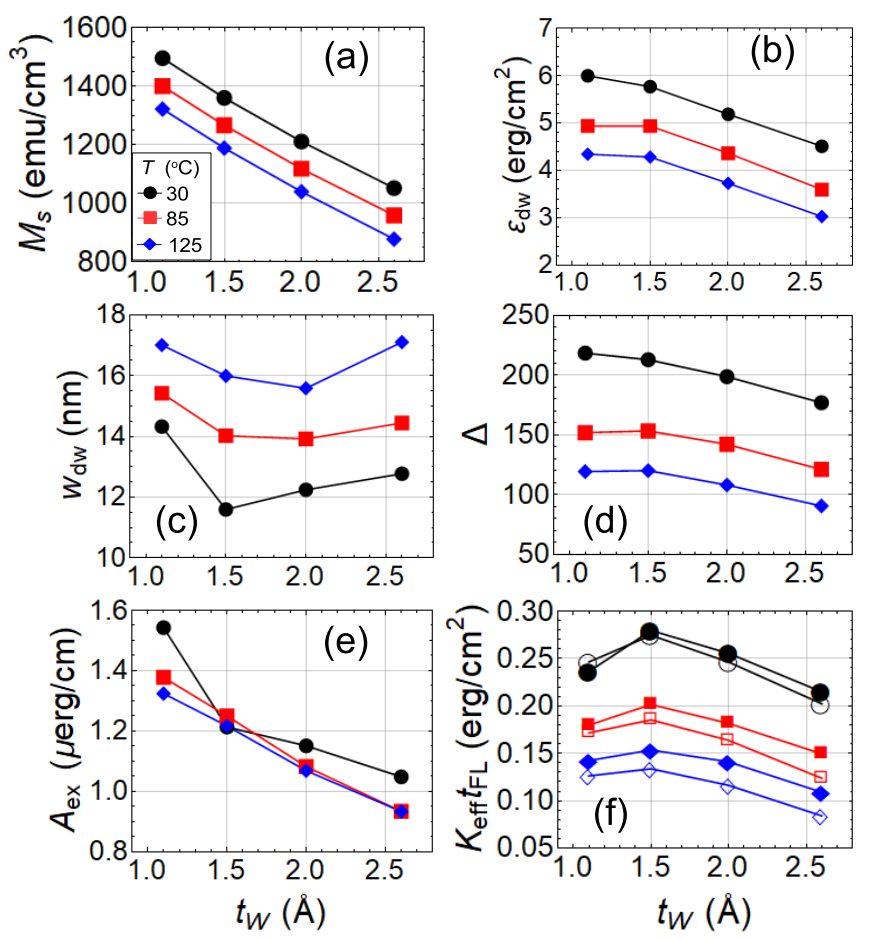

FIG. 4. (a) $M_{s}$, (b) $\varepsilon_{\mathrm{dw}}$, (c) $w_{\mathrm{dw}}$, (d) $\Delta$, (e) $A_{\mathrm{ex}}$ and (f) $K_{\mathrm{eff}} t_{\mathrm{FL}}=$ $(\ln 2 / 2)\left(\varepsilon_{\mathrm{dw}} / w_{\mathrm{dw}}\right) t_{\mathrm{FL}}$ (full symbols) and $K_{\mathrm{eff}} t_{\mathrm{FL}}=M_{S} H_{c} t_{\mathrm{FL}} / 2$ (open symbols) as a function of thickness of $\mathrm{W}$ insertion layers for $T=30$, 85 and $125{ }^{\circ} \mathrm{C}$. The legend shown in part (a) of the Figure refers to other parts as well. Each data point in (b)-(e) is an average value over $\mathrm{CD}=65,90,105$ and $120 \mathrm{~nm}$.

mate $\Delta$ from $K_{\text {eff }} t_{\mathrm{FL}}$ and $A_{\mathrm{ex}}$ measured on FL films 16 . Lower than expected values of $A_{\mathrm{ex}}$ in the former case and $\Delta$ in the latter case both can be explained by our finding that $\Delta$ is more directly determined by cell-level $H_{c}$ rather than film-level $H_{k}$ of the FL.

We further perform the same study at higher $T=85^{\circ} \mathrm{C}$ and $125{ }^{\circ} \mathrm{C}$. Fig. 4 summarizes our $T$-dependent results. While $M_{s}, \varepsilon_{\mathrm{dw}}, \Delta$ and $A_{\mathrm{ex}}$ decrease monotonically with increasing $t_{\mathrm{W}}$ at all $T$ s (see Figs. 4(a), 4(b), 4(d), and 4(e), respectively), $w_{\mathrm{dw}}$ (see Fig. 4(c)) and $K_{\text {eff }} t_{\text {FL }}$ (see Fig. 4(f)), show non-monotonic dependence on $t_{\mathrm{W}}$. In addition, one can see that $K_{\mathrm{eff}} t_{\mathrm{FL}}$ values calculated from the obtained $\varepsilon_{\mathrm{dw}}$ and $w_{\mathrm{dw}}$ (filled symbols) agree well with those obtained from $M_{s} H_{c}$ product (open symbols) for all $T \mathrm{~s}$, although for higher $T \mathrm{~s}$ the latter values tend to be smaller. This discrepancy increases with increasing $T$. Our numerical modeling shows that this behavior is expected, as the ratio $\xi=M_{s} H_{c} /\left(\varepsilon_{\mathrm{dw}} \ln 2 / w_{\mathrm{dw}}\right)$ has no universal value, but is a monotonically increasing function of $H_{c}$ : $\xi \cong 1$ for $H_{c}=2-2.5 \mathrm{kOe}$ (corresponding to values in our experiments at $T=30^{\circ} \mathrm{C}$, see Fig. 3(e)), but decreasing for lower values $H_{c}<2$ kOe that we measure at higher $T$ s (see Section III and Figs 5 and 6 of the Supplementary Material).

Our results show that thinner W IL could be advantageous for producing high thermal stability $\Delta$, despite having weaker PMA energy density $K_{\text {eff }} t_{\mathrm{FL}}$. The advantage stems from higher $M_{s}$ and $A_{e x}$ of the FL film, as well as weaker $T$ dependence of the latter parameters. Our results agree with film level studies presented in References ${ }^{2|15| 16}$.

In conclusion, we report an analytical model for calculating energy barrier for domain wall mediated magnetization reversal of the MRAM cell which describes correction to the droplet model previously used in literature. Using our model, we study thermal stability factor $\Delta$ for various thicknesses of $\mathrm{W}$ layers inserted into FL as a function of device size and temperature. We find that, by increasing $\mathrm{W}$ thickness, the effective PMA energy density of the FL film monotonically increases, but at the same time, $\Delta$ of the cell decreases. Our analysis shows that in order to maximize $\Delta$ for DWMR, one has to maximize $M_{s}$ and $A_{\text {ex }}$ of the FL film, and $H_{c}$ of the cell FL. Our results also show that thinner W IL could be advantageous for producing high $\Delta$ for MRAM cell sizes down to $\approx 25 \mathrm{~nm}$.

${ }^{1}$ W. F. Brown, Phys. Rev. 130, 1677 (1963).

${ }^{2}$ A. Meo, P. Chureemart, S. Wang, R. Chepulskyy, D. Apalkov, R. W. Chantrell, and R. F. L. Evans, Sci. Rep. 7, 16729 (2017).

${ }^{3}$ L. Thomas, G. Jan, S. Le, and P.-K. Wang, Appl. Phys. Lett. 106, 162402 (2015).

${ }^{4}$ L. Tillie, E. Nowak, R. C. Sousa, M.-C. Cyrille, B. Delaet, T. Magis, A. Persico, J. Langer, B. Ocker, I.-L. Prejbeanu, and L. Perniola, 2016 IEEE International Electron Devices Meeting (IEDM) , 27.3.1 (2015).

${ }^{5}$ L. Thomas, G. Jan, S. Le, Y.-J. Lee, H. Liu, J. Zhu, S. Serrano-Guisan, R.-Y. Tong, K. Pi, D. Shen, R. He, J. Haq, Z. Teng, A. Rao, V. Lam, Y.J. Wang, T. Zhong, T. Torng, and P.-K. Wang, 2015 IEEE International Electron Devices Meeting (IEDM), 26.4.1 (2015).

${ }^{6}$ C. Yoshida, T. Tanaka, T. Ataka, J. Fujisaki, K. Shimizu, T. Hirahara, and H. Shitara, J. J. Appl. Phys. 58, SBBB05 (2019).

${ }^{7}$ A. Meo, R. Chepulskyy, D. Apalkov, R. W. Chantrell, and R. F. L. Evans, arXiv, 1912.09761v1 (2019).

${ }^{8}$ H. Sato, M. Yamanouchi, S. Ikeda, S. Fukami, F. Matsukura, and H. Ohno, Appl. Phys. Lett. 101, 022414 (2012).

${ }^{9}$ G. Jan, Y.-J. Wang, T. Moriyama, Y.-J. Lee, M. Lin, T. Zhong, R.-Y. Tong, T. Torng, and P.-K. Wang, Appl. Phys. Express 5, 093008 (2012).

${ }^{10}$ S. Couet, T. Devolder, J. Swerts, S. Mertens, T. Lin, E. Liu, S. Van Elshocht, and G. Sankar Kar, Appl. Phys. Lett. 111, 152406 (2017).

${ }^{11}$ H. Almasi, D. Reifsnyder Hickey, T. Newhouse-Illige, M. Xu, M. R. Rosales, S. Nahar, J. T. Held, K. A. Mkhoyan, and W. G. Wang, App. Phys. Lett. 106, 182406 (2015).

${ }^{12} \mathrm{H}$. Almasi, M. Xu, J. Xu, T. Newhouse-Illige, and W. G. Wang, App. Phys. Lett. 109, 032401 (2016).

${ }^{13}$ J.-H. Kim, J.-B. Lee, G.-G. An, S.-M. Yang, W.-S. Chung, H.-S. Park, and J.-P. Hong, Sci. Rep. 5, 16903 (2015).

${ }^{14}$ J. M. Iwata-Harms, G. Jan, H. Liu, S. Serrano-Guisan, J. Zhu, L. Thomas, R.-Y. Tong, P. Sahil, V. Sundar, and P.-K. Wang, Sci. Rep. 8, 14409 (2018).

${ }^{15}$ J. B. Mohammadi, B. Kardasz, G. Wolf, Y. Chen, M. Pinarbasi, and A. K. Kent, ACS Appl. Electron. Mater. 1, 2025 (2019).

${ }^{16}$ J. M. Iwata-Harms, G. Jan, S. Serrano-Guisan, L. Thomas, H. Liu, J. Zhu, Y.-J. Lee, S. Le, R.-Y. Tong, P. Sahil, V. Sundar, D. Shen, Y. Yang, R. He, J. Haq, Z. Teng, V. Lam, P. Liu, Y.-J. Wang, T. Zhong, H. Fukuzawa, and P.-K. Wang, Sci. Rep. 9, 19407 (2019).

${ }^{17}$ D. Hinzke and U. Nowak, Phys. Rev. B 58, 265 (1998).

${ }^{18}$ G. D. Chaves-O'Flynn, G. Wolf, J. Z. Sun, and A. D. Kent, Phys. Rev. Appl. 4, 024010 (2015).

${ }^{19}$ D. C. Worledge and P. L. Trouilloud, App. Phys. Lett. 83, 84 (2003).

${ }^{20}$ M. Yamanouchi, A. Jander, P. Dhagat, S. Ikeda, F. Matsukura, and H. Ohno, IEEE Magn. Lett. 2, 3000304 (2011).

${ }^{21}$ B. Buford, P. Dhagat, and A. Jander, IEEE Magn. Lett. 7, 3107903 (2016). 
Supplementary Material for

\title{
Thermal stability for domain wall mediated magnetization reversal in perpendicular STT MRAM cells with $W$ insertion layers
}

\author{
G. Mihajlović ${ }^{1}$, N. Smith ${ }^{1 *}$, T. Santos ${ }^{1}$, J. Li ${ }^{1}$, B. D. Terris ${ }^{1}$ and J. A. Katine ${ }^{1}$ \\ ${ }^{1}$ Western Digital Research Center, Western Digital Corporation, San Jose, CA, 95119
}

*Corresponding author. Email: neil.smith@wdc.com

Section I. Fig. 1a shows the geometry of the "droplet" domain-wall model ${ }^{[1-4]}$ in the case of infinitesimal domain wall width. This model imposes the geometric constraint that the curved wall intersects the perimeter at a right angle. The area $A_{d}$ of the (smaller) domain is obtained by subtracting areas $A_{\text {green }}$ and $A_{\text {blue }}$ from right triangle $\frac{1}{2} r R$

$$
\begin{gathered}
\frac{1}{2} r R=\frac{1}{2} A_{d}+A_{\text {green }}+A_{\text {blue }} \\
\frac{1}{2} r^{2} \phi=\frac{1}{2} A_{d}+A_{\text {blue }}, \phi=\frac{\pi}{2}-\theta \\
\frac{1}{2} R^{2} \theta=\frac{1}{2} A_{d}+A_{\text {green }}, r=R \tan \theta
\end{gathered}
$$

Combining these results yields the following:

$$
\begin{gathered}
A_{d}=\frac{1}{4} D^{2}\left[\theta-\tan \theta+\left(\frac{\pi}{2}-\theta\right) \tan ^{2} \theta\right] \\
L_{\mathrm{dw}}=2 r \phi=D\left(\frac{\pi}{2}-\theta\right) \tan \theta
\end{gathered}
$$

$L_{\mathrm{dw}}$ being the domain wall arc length. Including the combination of domain wall energy $L_{\mathrm{dw}} t \varepsilon_{\mathrm{dw}}$ (where $\varepsilon_{\mathrm{dw}}$ is the domain-wall energy density, and $t$ the free-layer film thickness), and Zeeman energy in the presence of a uniform

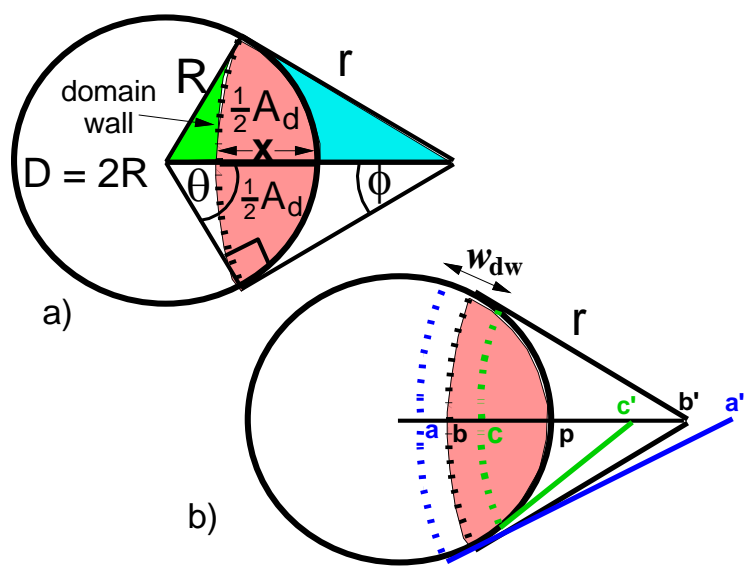

Fig. 1. (a) Droplet model with new length parameter $x$. (b) geometric illustration of corrected finite domain-wall width model. external (perpendicular) field $H$, the energy $E$ of the freelayer is expressed as

$$
E=L_{\mathrm{dw}} t \varepsilon_{\mathrm{dw}}+|H| M_{s} t\left[\frac{\pi}{4} D^{2}-A_{d}-A_{d}\right]
$$

$A_{d}$ is the area of the domain whose magnetization is parallel to the direction of field $H$. $\left(\frac{\pi}{4} D^{2}-A_{d}\right.$ is the area of the other domain.) It then follows that

$$
\begin{gathered}
E_{\max }=E\left(\theta_{0}\right),\left.\frac{d E}{d \theta}\right|_{\theta_{0}}=0 \\
d A_{d} / d \theta=\frac{1}{2} D \tan \theta d L_{d \mathrm{w}} / d \theta(\text { from (1)) } \\
\rightarrow \tan \theta_{0}=\varepsilon_{\mathrm{dw}} /\left(|H| M_{s} D\right)
\end{gathered}
$$

Finally, the energy barrier $E_{b}$ for domain-wall reversal is then

$$
E_{b}(H)=E\left(\theta_{0}\right)-\frac{\pi}{4} D^{2} M_{s} t H
$$

The latter reference term in (4) is the Zeeman energy of the initial uniform magnetization state of the free-layer. Here, a positive $H$ corresponds to a magnetic field that is antiparallel to the initial magnetization direction. The result in (4) implicitly assumes the $t / D \rightarrow 0$ limit so demagnetizing fields are local, and so the anisotropy energy does not depend on the position of the (zero wall-width) domain wall.

Unlike the macrospin model where $E_{b} \rightarrow 0$ at finite $H \rightarrow$ $H_{k \perp}$, the "droplet" model of [1-4] predicts that $E_{b} \rightarrow 0$ in the limit $H \rightarrow \infty$. This unphysical feature may be eliminated by inclusion of a small but finite domain-wall width $w_{\mathrm{dw}}$, as shown previously. ${ }^{[4]}$ This was implemented by reducing the area of both domains by modulating the wall position (see Fig. 1b) by an amount $\pm w_{\mathrm{dw}} / 2=|a-b|=|b-c|$ from original position $b$ (with $r=\left|b-b^{\prime}\right|$ ) while maintaining the aforementioned right-angle constraint at the perimeter. Using $r=r(\theta)=R \tan \theta$ as the metric, (2) is re-expressed as

$$
\begin{array}{r}
E(r)=L_{\mathrm{dw}}(r) t \varepsilon_{\mathrm{dw}}+|H| M_{s} t\left[\frac{\pi}{4} D^{2}-A_{d}\left(r+\frac{1}{2} w_{\mathrm{dw}}\right)\right. \\
\left.-A_{d}\left(r-\frac{1}{2} w_{\mathrm{dw}}\right)\right] .
\end{array}
$$


However, there is a geometric error in (5). Modulation $\pm w_{\mathrm{dw}} / 2$ in wall position is not equal to the modulation $\pm \Delta r$ in $r$ since the position of points $a^{\prime}, b^{\prime}$, and $c^{\prime}$ simultaneously vary, e.g., $+\Delta r=\left|a-a^{\prime}\right|-\left|b-b^{\prime}\right|>|a-b|=+w_{\mathrm{dw}} / 2$, e.g., see Fig. $1 \mathrm{~b}$. The error here is first order in $w_{\mathrm{dw}} / D$.

At the cost of some trigonometric complexity, this problem can be solved by using length $x$ (Fig. 1a) as the metric for wall position, since it is referenced to the fixed point $p$. Here, $+\Delta x=|a-p|-|b-p|=|a-b|=+w_{\mathrm{dw}} / 2$, exactly.

Geometrically, $x \equiv R+r-\sqrt{R^{2}+r^{2}}$, but it is here preferred to work with the dimensionless variable $q \equiv x / R$. Given that $r=R \tan \theta$, the relationship $\theta(q)$ is readily shown to be

$$
\begin{gathered}
\theta(0 \leq q<1)=\tan ^{-1}\left(\frac{q(1-q / 2)}{1-q}\right) \\
\theta(1<q<2)=\pi-\tan ^{-1}\left(\frac{q(1-q / 2)}{q-1}\right)
\end{gathered}
$$

Defining $\delta \equiv w_{\mathrm{dw}} / D$, the corrected expression for $E(q)$ replaces (5) with

$$
\begin{array}{r}
E(q)=L_{\mathrm{dw}}(q) t \varepsilon_{\mathrm{dw}}+|H| M_{s} t\left[\frac{\pi}{4} D^{2}-A_{d}(q+\delta)\right. \\
\left.-A_{d}(q-\delta)\right]
\end{array}
$$

It is understood that $A_{d}(q \pm \delta)=\left.A_{d}\left(\theta\left(q^{\prime}\right)\right)\right|_{q^{\prime}=q \pm \delta}$. The case $\theta\left(q^{\prime}>1\right)$ in (6) may arise in (7) when evaluating $A_{d}(q+\delta)$ using (1a) for "small" $|H|$ and "large" $\delta$.

Because $L_{\mathrm{dw}}(q)$ and $A_{d}(q \pm \delta)$ in (7) are evaluated for different arguments, the method of (3) cannot be used to obtain an exact solution for $q_{0}$ such that $\left.\frac{d E}{d q}\right|_{q_{0}}=0$. However, one can use (3c) and (6) to obtain an approximate solution: $q_{01}=1+\tan \theta_{0}-\sqrt{1+\tan ^{2} \theta_{0}}, \tan \theta_{0}=\varepsilon_{\mathrm{dw}} /\left(|H| M_{s} D\right)$, that is accurate to first order in $\delta$. The energy barrier can then be estimated to be

$$
E_{b}(H ; \delta) \approx E\left(q_{01}\right)-\frac{\pi}{4} D^{2} M_{s} t H
$$

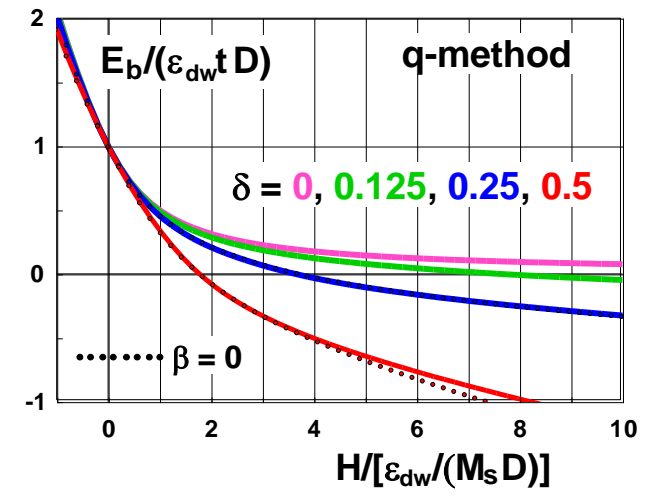

Fig. 2. Normalized $E_{b}(H ; \delta)$ for the q-method for indicated $\delta$. Dotted lines are for results $(\delta=0.25,0.5)$ without 2 nd order correction (9). using (7) to evaluate $E\left(q_{01}\right)$. Implicitly assumed here is that the wall core has zero net contribution to Zeeman energy. If $\delta>q_{01}, A_{d}\left(q_{01}-\delta\right)$ in (7) is taken to be zero.

A second order accurate solution $q_{02}=q_{01}+\beta \delta^{2}$ may be found by Taylor expanding $\frac{d E}{d q}=0$ from (7) to order $\delta^{2}$ using (3b), and substituting $q=q_{01}+\beta \delta^{2}$. One finds:

$$
\begin{gathered}
\beta=-\frac{\partial^{1} T_{q_{01}} \partial^{2} L_{q_{01}}+\frac{1}{2} T_{0} \partial^{3} L_{q_{01}}+\frac{1}{2} \partial^{2} T_{q_{01}} \partial^{1} L_{q_{01}}}{\partial^{1} T_{q} \partial^{1} L_{q}} \\
T_{0}=\frac{\varepsilon_{\mathrm{dw}}}{|H| M_{s} D^{\prime}}, T_{q}=\frac{q(1-q / 2)}{1-q}, \partial^{1} T_{q}=\frac{1}{2}\left[1+\frac{1}{(1-q)^{2}}\right] \\
\partial^{1} L_{q}=\frac{d L_{\mathrm{dw}}}{d q}=D\left[\frac{\pi}{2}-\theta(q)-\frac{T_{q}}{1+T_{q}^{2}}\right] \cdot \partial^{1} T_{q}
\end{gathered}
$$

using notation $\partial^{n} F_{q^{\prime}}=d^{n} F(q) /\left.d q^{n}\right|_{q=q^{\prime}}$. The explicit expression for $\beta$ is obviously quite cumbersome. The results from (8), with or without the $\beta$-correction from (9), will be referred to below as the " $q$-method".

Fig. 2 shows normalized $E_{b}(H ; \delta)$ via the $q$-method with varied $\delta \equiv w_{\mathrm{dw}} / D$. As referred to earlier, $E_{b}$ remains finite for all $H$ when $\delta \rightarrow 0$. Even for "large" $\delta=0.5$ (beyond which the model itself becomes questionable), the $2^{\text {nd }}$ order $\beta$-correction has minimal impact on $E_{b}$, particularly in the $\mathrm{H}$ range of most interest where $E_{b}>0$. The primary reason is that $E_{b}$ is evaluated at the energy maximum, making its value insensitive to more finite differences between $q_{01}$ and $q_{02}$. However, it is only $E_{b}(H ; \delta)$ that plays a role when fitting field-switching probability data.

If one returns to (5), and defines $p \equiv r / R=\tan \theta$, one can rewrite it in a form analogous to (7):

$$
\begin{gathered}
E(p)=L_{\mathrm{dw}}(p) t \varepsilon_{\mathrm{dw}}+|H| M_{s} t\left[\frac{\pi}{4} D^{2}-A_{d}(p+\delta)\right. \\
\left.-A_{d}(p-\delta)\right](10 \mathrm{a}) \\
E_{b}(H ; \delta) \approx E\left(p_{0}\right)-\frac{\pi}{4} D^{2} M_{s} t H,
\end{gathered}
$$

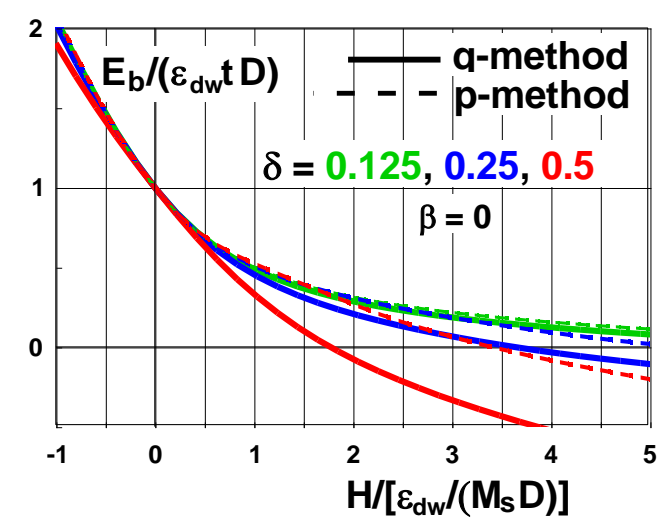

Fig. 3. Normalized $E_{b}(H ; \delta)$ comparing p-method (dashed) with 1 st-order q-method (solid) for indicated $\delta$. 


$$
\theta_{0}=\tan ^{-1}\left(p_{0}=\frac{\varepsilon_{\mathrm{dw}}}{|H| M_{s} D}\right)
$$

The results of (10) will be referred to as the " $p$-method", the equivalent of Ref. [4]. It is only exact to zeroth order in $\delta$. Unlike the $q$-method, the $p$-method is ill defined when field $H \rightarrow 0$ and $\delta>0$, and some "care" is required in that case.

Fig. 3 shows a comparison of $q$-method and $p$-method computations of normalized $E_{b}(H ; \delta)$. Not unexpectedly, the $p$-method suffers serious error for $\delta \gtrsim 0.25$, where the $p$ method overestimates by roughly a factor of 2 the value of $\delta$ that will most closely reproduce the $E_{b}(H ; \delta)$ curves generated by the more exact $q$-method.

Section II. The domain-wall width parameter $w_{\mathrm{dw}}$ in (5) or (7) is used to quantify the loss in Zeeman energy with a sharp wall of finite thickness when abandoning the zero-thickness domain wall of the original droplet model. To relate $w_{\mathrm{dw}}$ to exchange stiffness $A$ and anisotropy constant $K_{\text {eff }}$ that characterize an analytic form of (Bloch) domain wall ${ }^{[5]}$, one can equate the loss of Zeeman energy using these two wall profiles $m_{z}(x)$. Since the Zeeman energy scales proportionately to $\int m_{z}(x) d x$, this comparison is expressed as

$$
\begin{gathered}
\int_{0}^{L}\left[1-m_{z}^{\text {sharp }}(x)\right] d x=\int_{0}^{L}\left[1-m_{z}^{\text {Bloch }}(x)\right] d x \\
\left.m_{z}^{\text {sharp }}(x)=0 \text { (if } x<w_{\mathrm{dw}} / 2\right) \text {, or } 1\left(\text { if } x>w_{\mathrm{dw}} / 2\right)
\end{gathered}
$$

$$
m_{z}^{\text {Bloch }}(x)=\tanh \left(\sqrt{K_{\text {eff }} / A} x\right)
$$

the " 1 " in the integrands of (11a) representing the zerothickness wall. Solving (11a) with $L \gg\left(w_{\mathrm{dw}} / 2, \sqrt{A / K_{\mathrm{eff}}}\right)$, one finds

$$
w_{\mathrm{dw}}=2 \ln 2 \sqrt{A / K_{\mathrm{eff}}}
$$

The result in (12) is similar to that proposed in Ref. [4]. It is notably distinct from the classical domain-wall width $\pi \sqrt{A / K_{\text {eff }}}$ based on extrapolation of the wall-profile shape $\mathrm{e}^{[5]}$, but which is not directly relevant to the Zeeman energy consideration of importance here.

Section III. Expressing the results illustrated in Fig. 2 as

$$
E_{b} / \varepsilon_{\mathrm{dw}} D t=F\left(H_{z} M_{s} D / \varepsilon_{\mathrm{dw}} ; \delta\right)
$$

with $F()$ defined through (7)-(9). For an Arrhenius model for field-switching probability $P_{\mathrm{sw}}=1-\exp \left(-f_{0} t_{\mathrm{sw}} e^{-\Delta}\right)$ in a time $t_{\mathrm{sw}}$, where $\Delta=E_{b} / k_{B} T$ and "attempt-frequency $f_{0} \sim$ $10^{9} \mathrm{sec}^{-1}$, the coercivity $H_{c}$ is the $H_{z}$ where $P_{\mathrm{sw}}=50 \%$, or $\Delta_{50 \%}=\ln \left(f_{0} t_{\mathrm{sw}} / \ln 2\right)$. Letting $H_{z} \rightarrow H_{c}$ and $\Delta \rightarrow \Delta_{50 \%}$, it follows from (13) that

$$
\varepsilon_{\mathrm{dw}} D t / k_{B} T F\left(H_{c} M_{s} D / \varepsilon_{\mathrm{dw}} ; \delta\right)=\Delta_{50 \%}
$$

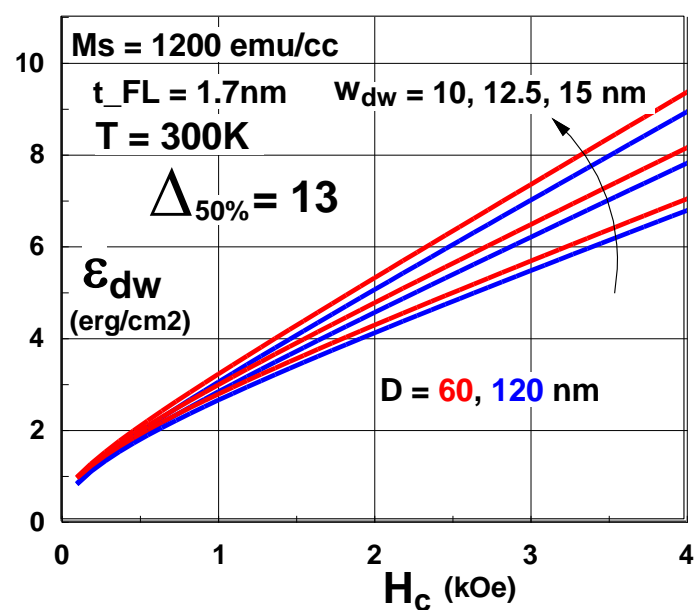

Fig. 4. Solutions of (14) for values of FL diameter $D$ and domain wall-width $w_{d w}$ (increasing in direction of arrow) as indicated.

Treating $\Delta_{50 \%}$ as a known constant, (14) can be solved numerically to obtain $\varepsilon_{\mathrm{dw}}$ as a function of $H_{c}$. Some examples for $\varepsilon_{\mathrm{dw}}\left(H_{c}\right)$ are shown in Fig. 4. The value of $\Delta_{50 \%} \cong 13$ corresponds to $t_{\mathrm{sw}} \sim 2 \mathrm{msec}$. The parameter value choices are relevant to the experimental conditions presented in our publication.

In terms of exchange stiffness $A$ and anisotropy constant $K_{\text {eff }}$, the domain wall energy density $\varepsilon_{\mathrm{dw}}=4 \sqrt{A K_{\mathrm{eff}}}$ for the aforementioned Bloch wall model. ${ }^{[5]}$ This, along with (12), can be used to solve for $K_{\mathrm{eff}}=\frac{1}{2} \ln 2 \varepsilon_{\mathrm{dw}} / w_{\mathrm{dw}}$. One can compare this value of $K_{\text {eff }}$ to that of a "quasi-macrospin" model $K_{\text {eff }}^{\prime}=\frac{1}{2} M_{s} H_{c}$ which replaces $H_{k \perp}^{\text {eff }}$ of the true macrospin model by the coercivity $H_{c}$. In Fig. 5, the solutions shown in Fig. 4 can be re-expressed as plots of $K_{\text {eff }}^{\prime} / K_{\text {eff }}$ vs $H_{c}$. Interestingly, this ratio is close to unity for parameter values relevant to the experimental conditions presented in our publication (e.g. see Fig. 6). However, this behavior is not universal, particularly for lower $H_{c}$.

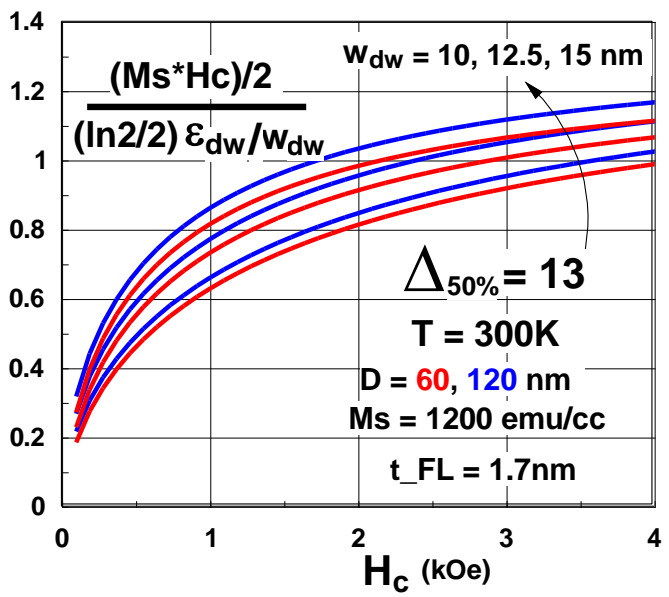

Fig. 5. The ratio $K_{\text {eff }}^{\prime} / K_{\text {eff }}$ vs $H_{c}$. Based on the solutions of Fig. 4. 


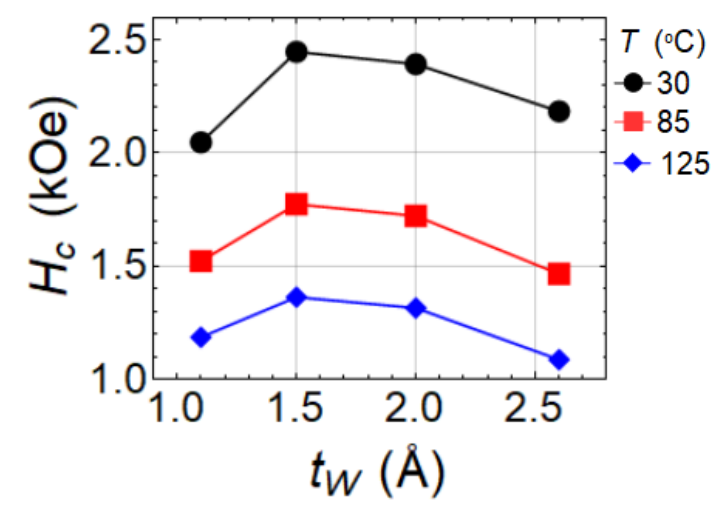

Fig. 6. $H_{c}$ vs W thickness at different Ts.

References:

[1] A. Meo, R. Chepulskyy, D. Apalkov, R. W. Chantrell, and R. F. L. Evans, arXiv , 1912.09761v1 (2019).

[2] D. Hinzke and U. Nowak, Phys. Rev. B 58, 265 (1998).

[3] G. D. Chaves-O'Flynn, G. Wolf, J. Z. Sun, and A. D. Kent, Phys. Rev. Appl. 4, 024010 (2015).

[4] L. Thomas, G. Jan, S. Le, Y.-J. Lee, H. Liu, J. Zhu, S. SerranoGuisan, R.-Y. Tong, K. Pi, D. Shen, R. He, J. Haq, Z. Teng, A.

Rao, V. Lam, Y.- J. Wang, T. Zhong, T. Torng, and P.-K. Wang, 2015 IEEE International Electron Devices Meeting (IEDM), 26.4.1 (2015).

[5] S. Chikazumi, Physics of Ferromagnetism, $2^{\text {nd }}$ Ed. Oxford University Press (1996). 